\title{
Drug Similarity Search Based on Combined Signatures in Gene Expression Profiles
}

\author{
Kihoon Cha, MS ${ }^{1}$, Min-Sung Kim, $\mathrm{PhD}^{2}$, Kimin Oh, $\mathrm{MS}^{1}$, Hyunjung Shin, $\mathrm{PhD}^{3}$, Gwan-Su Yi, $\mathrm{PhD}^{1}$ \\ Departments of ${ }^{1} \mathrm{Bio}$ and Brain Engineering and ${ }^{2}$ Information and Communications Engineering, Korea Advanced Institute of Science and Technology, Dae- \\ jeon; ${ }^{3}$ Department of Industrial Engineering, Ajou University, Suwon, Korea
}

Objectives: Recently, comparison of drug responses on gene expression has been a major approach to identifying the functional similarity of drugs. Previous studies have mostly focused on a single feature, the expression differences of individual genes. We provide a more robust and accurate method to compare the functional similarity of drugs by diversifying the features of comparison in gene expression and considering the sample dependent variations. Methods: For differentially expressed gene measurement, we modified the conventional $t$-test to normalize variations in diverse experimental conditions of individual samples. To extract significant differentially co-expressed gene modules, we searched maximal cliques among the co-expressed gene network. Finally, we calculated a combined similarity score by averaging the two scaled scores from the above two measurements. Results: This method shows significant performance improvement in comparison to other approaches in the test with Connectivity Map data. In the test to find the drugs based on their own expression profiles with leave-one-out cross validation, the proposed method showed an area under the curve (AUC) score of 0.99, which is much higher than scores obtained with previous methods, ranging from 0.71 to 0.93 . In the drug networks, we could find well clustered drugs having the same target proteins and novel relations among drugs implying the possibility of drug repurposing. Conclusions: Inclusion of the features of a co-expressed module provides more implications to infer drug action. We propose that this method be used to find collaborative cellular mechanisms associated with drug action and to simply identify drugs having similar responses.

Keywords: Pharmacological Biomarkers, Transcriptome, Gene Expression Regulation, Gene Regulatory Networks, Drug Repositioning

Submitted: November 15, 2013

Revised: January 24, 2014

Accepted: January 27, 2014

\section{Corresponding Author}

Gwan-Su Yi, PhD

Department of Bio and Brain Engineering, Korea Advanced Institute of Science and Technology, 291, Daehak-ro, Yuseong-gu, Daejeon 305-701, Korea. Tel: +82-42-350-6160, Fax: +82-42-350-6814, Email: gsyi@kaist.ac.kr

This is an Open Access article distributed under the terms of the Creative Commons Attribution Non-Commercial License (http://creativecommons.org/licenses/bync/3.0/) which permits unrestricted non-commercial use, distribution, and reproduction in any medium, provided the original work is properly cited.

(C) 2014 The Korean Society of Medical Informatics

\section{Introduction}

A gene expression profile provides a snapshot of the transcriptional level in a cell under specific conditions. It is widely used in biological analyses, including identification of genes that show expression patterns associated with certain phenotypes, identification of molecular markers that can be used to diagnose a disease state, and inference of the regulatory relationships of genes corresponding to a biological process [1]. The representative way to analyze an expression profile is to identify differentially expressed genes (DEGs) or co-expressed genes related to certain phenotypes. In ad- 
dition, researchers can also compare expression patterns of profiles with those of other profiles. This comparison can provide an alternative way to query large-scale gene expression repositories, such as the Gene Expression Omnibus and ArrayExpress, in a content based manner [2-5]. To support comparison of expression patterns, various expression similarity search methods have been devised.

Since the advent of the Connectivity Map [6], which provides large-scale libraries of expression profiles corresponding to human cell lines treated with diverse small molecules, the usage of expression similarity search has been extended to drug discovery and development. The Connectivity Map also provides a comparison method to measure similarities between a query gene signature and these reference profiles. To measure the similarity, a query gene signature comprising up- and down-regulated genes from the user is required. The Connectivity Map calculates the score of each reference profile by estimating whether the up-regulated genes exist at the top of the reference profile and whether the down-regulated genes exist at the bottom of the reference profile. The usefulness of the dataset and comparison approach is proved by the performance of case studies related to drug repurposing, unveiling of the mechanism of action of uncharacterized small molecules, the mimicry of a certain biological state, etc. This innovative approach has led to the development of diverse methods to large-scale analysis of drug response profiles [7-12]. Although these variant methods have adopted different pattern matching approaches to measure similarity, the major concept of similarity measurement, which is the use of the up- and down-regulated gene signature from the query profile, is still commonly used.

Co-expressed gene sets can be identified from drug response profiles using clustering methods [13]. In clustering, it is assumed that the expression patterns of co-expressed gene sets are correlated in all conditions. Alternatively, biclustering can be used to search gene sets that are correlated in a subset of the conditions [14]. Significantly perturbed or newly generated co-expressed gene sets from a drug response profile can be more appropriate to explain the expression states of cells treated with a certain drug. Several studies have identified differentially co-expressed genes known to be involved in cancer, even though their expression levels were not significantly changed [15-17]. For such reasons, genes with differential co-expression patterns can be another valuable signature to characterize drug treatment profiles in addition to DEGs.

In this paper, we propose a novel method to measure the similarities between drug response profiles by combining DEGs and dilated cardiomyopathy (DCMs) simultaneously.
Our method shows significant performance improvement in comparison to other approaches in a test with Connectivity Map data. We construct a drug-drug network using our method and find well clustered drugs having the same target proteins. Using the proposed method, drug-associated collaborative cellular mechanisms can be identified as well as drugs having similar gene expression responses.

\section{Methods}

\section{Construction of Drug Dataset}

The drug response data was downloaded from the Connectivity Map website (http://www.broadinstitute.org/cmap/). It contains 6,100 gene expression profiles of 5 cell lines treated with 1,309 distinct small molecules. The expression profiles were grouped by batch ID, and then each group was normalized by robust multi-array average. After the extraction of expression values from each experiment, they were merged into a single matrix. The merged expression profiles were further processed with limma package to normalize the expression intensities between different arrays using $\mathrm{R}$ [18].

Among the 5 cell lines, we used 3 cell lines (PC3, human prostate cancer cell lines; MCF7, human breast adenocarcinoma cell line; HL60, human promyelocytic leukemia cells) for this study because only a few experiments had been performed with the other two cell lines (SKMEL5, ssMCF7). The small molecules measured at least 10 times were selected for this research, and 29 target drugs passed this criterion. After the selection, we collected pharmaceutical information on the 29 selected drugs from drug databases. The drug information includes target proteins, the Anatomical Therapeutic Chemical classification code, and drug indication as listed in Table 1.

As a final step, we randomly selected 10 experiments from each drug to reduce bias by the number of experiments. For instance, trichostatin A was experimented with 181 times. In contrast, metformin was measured only 10 times.

\section{Construction of Previous Differential Expression Simi- larity Search Methods}

For gene-level similarity analysis, 5 different methods were implemented. First, the differential expression profiles of all genes were compared by using Spearman rank correlation (CellMontage) [4]. The other 4 methods used gene signatures, which comprise a preselected number of top/down DEGs, extracted only from the query profile. The Connectivity Map uses Kolmogorov-Smirnov statistics to measure the correlation pattern of two drug response profiles. Iorio et al. [8] measured the Gene Set Enrichment Analysis score. 
Cheng et al. [11] calculated the cosine similarity between two differential expression profiles that can be calculated as a dot product of two vectors. Zhang and Gant [7] devised a method that orders genes based on their absolute value of differential expression. Based on the ranking, the importance of a gene is allocated by the absolute value of its rank rather than its differential expression value. The direction of regulation is maintained by additionally allocating importance with the sign of its rank. Based on the reassigned query gene signature and reference expression profile, the connection strength between them is calculated.

\section{Identification of Differentially Co-expressed Gene Module Signature}

A co-expressed gene module, a group of genes, shows correlated expression pattern in all samples. In comparison with the module, genes consisting of DCMs do not need to be correlated across all conditions. The genes only need to be correlated in one of two predefined conditions: control or drug treatment. Therefore, we can identify two types of DCMs. A down-correlation module is a gene group which shows correlation in control samples, and the correlation completely disappears or is significantly reduced after drug

Table 1. Pharmaceutical information of 29 selected small molecules

\begin{tabular}{lllc}
\hline \multicolumn{1}{c}{ Drug name } & \multicolumn{1}{c}{ Indication } & \multicolumn{1}{c}{ Target protein } & ATC code \\
\hline Trichostatin A & Cancers & Histone deacetylase (inhibitor) & N/A \\
Vorinostat & Cutaneous T-cell lymphoma & Histone deacetylase (inhibitor) & L01XX38 \\
Valproic acid & Anticonvulsant & Histone deacetylase (inhibitor), histone & N03AG01 \\
& & acetyltransferase (inhibitor), GABA & aminotransferase (inhibitor), calcium \\
& & channel T type (blocker), short/branched & chain specific acyl-CoA dehydrogenase \\
& & (inhibitor)
\end{tabular}

$\begin{array}{ll}\text { LY-294002 } & \text { Cancer } \\ \text { Wortmannin } & \text { Hematologic malignancies }\end{array}$

\begin{tabular}{|c|c|}
\hline Sirolimus & Organ rejection \\
\hline Fulvestrant & Breast cancer \\
\hline Estradiol & $\begin{array}{l}\text { Urogenital symptoms associated with } \\
\text { post-menopausal atrophy of the vagina } \\
\text { and/or the lower urinary tract }\end{array}$ \\
\hline Haloperidol & Antipsychotic \\
\hline
\end{tabular}

Prochlorperazine

Psychotic disorders, nonpsychotic anxiety; severe nausea and vomiting

Clozapine Treatment-resistant schizophrenia

Chlorpromazine

Schizophrenia; nausea and vomiting; restlessness and apprehension; tetanus; manic-depressive illness
Phosphoinositide-3-kinases (inhibitor)

Phosphatidylinositol 3-kinase (inhibitor), phosphatidylinositol-4,5-bisphosphate 3-kinase catalytic subunit, delta isoform (inhibitor)

Serine/threonine-protein kinase mTOR L04AA10 (inhibitor), FK506-binding protein 1A, heparin-binding growth factor 2

Estrogen receptor (antagonist)

L02BA03

Estrogen receptor (agonist), orphan nu- G03CA01, G03CA03, clear receptor PXR (unknown)

L02AA02, L02AA03

Dopamine receptor (antagonist), gluta-

N05AD01 mate [NMDA] receptor subunit epsilon-2 (antagonist), 5-HT2A receptor

Dopamine receptor (antagonist)

N05AB04

5-HT receptor (antagonist), alpha1N05AH02 adrenergic receptor (antagonist), dopamine receptor (antagonist)

5-HT2 receptor (antagonist), H1 recep-

N05AA01 tor (antagonist), alpha2-adrenergic receptor (antagonist), dopamine receptor (antagonist), muscarinic cholinergic receptor (antagonist) 
Table 1. Continued

\begin{tabular}{|c|c|c|c|}
\hline Drug name & Indication & Target protein & ATC code \\
\hline Fluphenazine & Psychotic disorders & Dopamine receptor (antagonist) & N05AB02 \\
\hline Trifluoperazine & $\begin{array}{l}\text { Anxiety disorders, depressive symptoms } \\
\text { secondary to anxiety and agitation }\end{array}$ & Dopamine receptor (antagonist) & N05AB06 \\
\hline Thioridazine & $\begin{array}{l}\text { Schizophrenia and generalized anxiety } \\
\text { disorder }\end{array}$ & Dopamine receptor (antagonist) & N05AC02 \\
\hline Tanespimycin & $\begin{array}{l}\text { Breast cancer, melanoma; multiple my- } \\
\text { eloma }\end{array}$ & Heat shock protein HSP90 (inhibitor) & - \\
\hline Geldanamycin & - & Heat shock protein HSP90 (inhibitor) & - \\
\hline Alvespimycin & $\begin{array}{l}\text { Ovarian cancer, refractory hematological } \\
\text { malignancies; breast cancer; leukaemia }\end{array}$ & Heat shock protein HSP90 (inhibitor) & - \\
\hline Monorden & - & Heat shock protein HSP90 (inhibitor) & - \\
\hline Alpha-estradiol & Hair loss & Androgen receptor (antagonist) & - \\
\hline Troglitazone & Type II diabetes mellitus & $\begin{array}{l}\text { Peroxisome proliferator-activated recep- } \\
\text { tor gamma (agonist) }\end{array}$ & A10BG01 \\
\hline Rosiglitazone & Type II diabetes mellitus & $\begin{array}{l}\text { Peroxisome proliferator-activated recep- } \\
\text { tor gamma (agonist) }\end{array}$ & A10BG02 \\
\hline Pioglitazone & Type II diabetes mellitus & $\begin{array}{l}\text { Peroxisome proliferator-activated recep- } \\
\text { tor gamma (agonist) }\end{array}$ & A10BG03 \\
\hline $\begin{array}{l}\text { Nordihydroguaiaretic } \\
\text { acid }\end{array}$ & - & $\begin{array}{l}\text { Acetyl-CoA acetyltransferase, mitochon- } \\
\text { drial (inhibitor), arachidonate 5-lipox- } \\
\text { ygenase (inhibitor) }\end{array}$ & - \\
\hline $\begin{array}{l}\text { 15-delta } \\
\text { prostaglandin } \mathrm{J} 2\end{array}$ & - & - & - \\
\hline Tretinoin & $\begin{array}{l}\text { Acute promyelocytic leukemia; photo- } \\
\text { damage }\end{array}$ & Retinoic acid receptor (agonist) & D10AD01, L01XX14 \\
\hline Acetylsalicylic acid & Various forms of pain, inflammation & $\begin{array}{l}\text { Cyclooxygenase-1 (inhibitor), cyclooxy- } \\
\text { genase-2 (inhibitor) }\end{array}$ & $\begin{array}{l}\text { A01AD05, B01AC06, } \\
\text { N02BA01 }\end{array}$ \\
\hline Metformin & NIDDM, polycystic ovary syndrome & AMPK (activator) & A10BA02 \\
\hline Genistein & Prostate cancer & $\begin{array}{l}\text { DNA topoisomerase } 2 \text {-alpha (inhibitor), } \\
\text { estrogen receptor (inhibitor), estrogen } \\
\text { receptor beta (inhibitor), peroxisome } \\
\text { proliferator activated receptor gamma } \\
\text { (agonist) }\end{array}$ & - \\
\hline
\end{tabular}

ATC code: the Anatomical Therapeutic Chemical classification code, GABA: gamma-aminobutyric acid, mTOR: mammalian target of rapamycin, PXR: pregnane X receptor, NMDA: N-methyl-D-aspartate receptor, NIDDM: non-insulin-dependent diabetes mellitus, AMPK: AMP-activated protein kinase.

treatment. In contrast, the correlation of certain genes, or an up-correlation module, can be newly generated after drug treatment. We identified DCMs which satisfy the following two conditions: 1) DCMs are composed of significantly coexpressed gene pairs in one of the two conditions and 2) the gene pairs consisting of identified modules show significant differential co-expression compared to the other condition.
The overall procedure to identify DCM signatures is illustrated in Figure 1. To identify a significant co-expressed gene pairs in the two conditions, we measured the Pearson correlation coefficient (PCC) of all possible combinations of gene pairs and used them to find the distribution of correlations in each condition. In the correlation distribution, we selected the top $5 \%$ of gene pairs in the distribution and defined them 


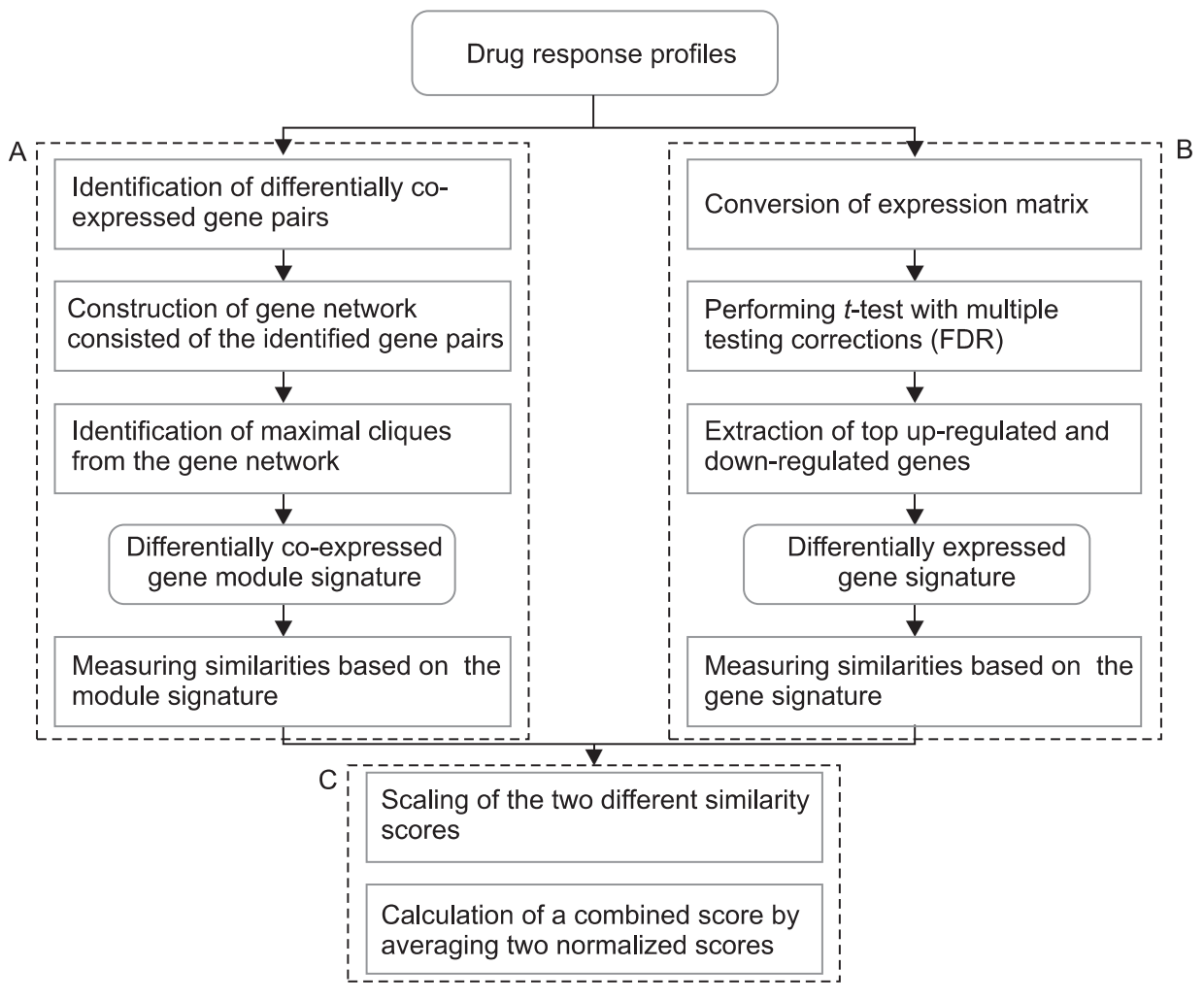

Figure 1. Overall procedures to measure differential expression similarity. (A) identification of differentially coexpressed gene module signature. (B) identification of differentially expressed gene signature. (C) Calculation combined score of the two different signatures. FDR: false discovery rate. as significantly co-expressed gene pairs. Among the significant gene pairs, we further selected gene pairs which showed significant differential co-expression. For the selection, we also found the distribution of differential co-expression by measuring all possible differences of co-expressions of gene pairs in the two conditions. The top $5 \%$ of the gene pairs were assumed to be significant differential co-expression pairs. The gene pairs that satisfied the two conditions were used to form the gene differential co-expression network. In the network, each gene pair was assumed to be a seed group, and each seed group is expanded by identifying the maximal clique in the network. A maximal clique is a sub-network, the nodes of which are fully connected with all other nodes.

The detailed expansion method was performed with the following procedures. If we found a down-correlation module, the seed groups were sorted based on the co-expression value in the control samples. Among the sorted seed groups, the group with the highest co-expression value was selected for expansion. Based on the selected seed group, we searched neighbor genes connected to all member genes of the seed group. The distance to a neighbor was measured by averaging the co-expression values between the neighbor and member genes in the group. Among the neighbors, the gene with the highest co-expression values was selected for merging. This merging step was iteratively performed until there was no neighbor gene that was fully linked to members of the currently expanded module. If the expansion of the selected seed group was finished, the genes included to the expanded module were removed from the sorted gene pairs. With the remaining gene pairs, the expansion of the next seed group was iteratively performed. The identified modules are maximal cliques in the gene co-expression network which comprise gene pairs that have significantly co-expressed in the target condition and have a higher differential co-expression value compared to the other condition. The up-correlation module is also searched with the same procedures by searching with co-expressed gene pairs in drug treatment samples. We set the minimum module size as 6 in this research.

\section{Identification of Differentially Expressed Gene Signature}

In the Connectivity Map, the transcriptional response of each drug is measured from independent experiments varying diverse conditions including different cell lines. Therefore, even though experiments are performed with the same drug, they have significantly different expression values in the control samples. The representative way to identify significantly DEGs in two different conditions is to perform a $t$-test. The $t$-test finds genes that have a slight variance of expression values in each condition and significant changes of expression values between the two conditions. The expression inconsistency of each gene in control samples can ham- 
per the identification of DEGs using the method. Therefore, we converted the expression value of each gene into a new value by adapting these simple formulas:

$$
\begin{aligned}
& N_{\text {Control }}=E_{\text {Control }}-\frac{E_{\text {Control }}+E_{\text {Drug }}}{2}, \\
& N_{\text {Drug }}=E_{\text {Drug }}-\frac{E_{\text {Control }}+E_{\text {Drug }}}{2} .
\end{aligned}
$$

Here, $E_{\text {Control }}$ and $E_{\text {Drug }}$ denote the original expression values of each drug response experiment. This simple conversion can reduce the inconsistency of expression values in control samples by shifting the original values and focusing more on the degree of differential expression values. By using the converted expression profiles, we performed a $t$-test with a false discovery rate (FDR) multiple testing corrections of 0.05 to the profiles. To identify significant DEGs, we extracted 100 up-regulated genes and 100 down-regulated genes with the lowest $p$-value.

\section{Differential Expression Similarity Search with Identi- fied Gene Signature}

We performed a similarity search between drug response profiles with the identified DCMs and DEGs. Before calculation of the combined score of the two different signatures, the differential expression similarity based on each signature was measured independently. For the calculation of the DCM-based similarity score, the modules were identified from the query profile. We calculated the differentially coexpressed gene module-based similarity score (DCS) between two profiles, namely, the query profile and reference profile:

$$
\begin{aligned}
& D C_{U P}=P C C_{\text {Control }}-P C C_{\text {Drug }}, \\
& D C_{D O W N}=P C C_{\text {Drug }}-P C C_{\text {Control }}, \\
& D C M=\sum_{i=1}^{n} \frac{D C_{i}^{\text {query }}-D C_{i}^{\text {ref }}}{n}, \\
& D C S=\sum_{j=1}^{N} \frac{D C M_{j}}{N} .
\end{aligned}
$$

Here, DC denotes the differential co-expression value of a gene pair in a certain condition. We calculated two different types of DCs because there are two different types of modules in the module signature, that is, an up-correlation module and down-correlation module. DCM denotes the similarity score of each module. In each module, there are $n$ possible combinations of gene pairs. For each gene pair, we calculated the difference between the DC from the query profile and the DC from the reference profile. The DCS was measured by averaging the score of all identified DCM signatures.

To calculate the DEG-based similarity score, $\operatorname{Sim}_{D E G}$, we retrieved DEGs from the query profile. We averaged the differential expression values of each selected gene because there are multiple instances in the query and reference profiles. Based on the DEGs, we measured the PCC between the query profile and reference profile. After calculating two similarity scores, $\operatorname{Sim}_{\text {Module }}$ and $\operatorname{Sim}_{D E G}$, we scaled them because they had different ranges of scores. We scaled each score between 0 and 1:

$$
\operatorname{NSim}_{k}=\frac{\operatorname{Sim}_{k}-\operatorname{Sim}_{\min }}{\operatorname{Sim}_{\mathrm{max}}-\operatorname{Sim}_{\mathrm{min}}} .
$$

The combined score of the two different signature-based similarity scores is defined as

$$
N \operatorname{Sim}_{\text {combined }}=\frac{\text { NSim }_{\text {Module }}+N \operatorname{Sim}_{D E G}}{2} .
$$

\section{Results}

\section{Performance Comparison of Differential Expression Similarity Search Methods}

We measured the performance of several similarity search methods by calculating the area under the receiver operating characteristic (ROC) curve, or the area under the curve (AUC). Figure 1 shows the overall performance of each method. For the performance estimation of previous similarity search methods, we used differential expression information from each instance. For the gene signature-based methods, we extracted 100 up-regulated genes and 100 downregulated genes from query instances to use them as gene signatures. After measuring the performance of previous methods, we found that there is no significant performance difference between previous similarity search methods that use differential expression information only from the input query profile. If a certain similarity search method shows good performance by allocating highly positive similarity scores between the expression profiles treated with the target drug, the other methods also show good performance for the target drug. If a certain method cannot effectively find similarities between instances measured with the target drug, the others also cannot find the connections between them. These results indicate that, although the development of an effective similarity measurement method is important, the extraction of appropriate response signatures from drug response profiles was more important.

To compare our method with previous differential expres- 
Table 2. Performance of expression similarity search methods for selected 29 drugs

\begin{tabular}{|c|c|c|c|c|c|c|}
\hline Drug name & CellMontage [4] & Cheng et al. [11] & Connectivity Map [6] & Zhang and Gant [7] & lorio et al. [8] & Combined score \\
\hline 15-delta prostaglandin $\mathrm{J} 2$ & 0.8953 & 0.9617 & 0.9836 & 0.9966 & 0.9993 & 1.0000 \\
\hline LY-294002 & 0.7304 & 0.8419 & 0.8731 & 0.8438 & 0.8941 & 0.9995 \\
\hline Acetylsalicylic acid & 0.5702 & 0.9141 & 0.8859 & 0.9568 & 0.9839 & 1.0000 \\
\hline Alpha-estradiol & 0.5814 & 0.9630 & 0.8927 & 0.9854 & 0.9989 & 1.0000 \\
\hline Alvespimycin & 0.9452 & 0.9938 & 0.9972 & 0.9991 & 1.0000 & 1.0000 \\
\hline Chlorpromazine & 0.5500 & 0.8172 & 0.7489 & 0.8355 & 0.8505 & 1.0000 \\
\hline Fulvestrant & 0.5390 & 0.7144 & 0.8657 & 0.7593 & 0.8157 & 1.0000 \\
\hline Geldanamycin & 0.9689 & 0.9942 & 0.9951 & 0.9997 & 1.0000 & 1.0000 \\
\hline Genistein & 0.6602 & 0.8518 & 0.9529 & 0.9067 & 0.9461 & 1.0000 \\
\hline Haloperidol & 0.5055 & 0.9233 & 0.9164 & 0.9769 & 0.9964 & 0.9999 \\
\hline Metformin & 0.5761 & 0.6901 & 0.6872 & 0.7336 & 0.8150 & 1.0000 \\
\hline Monorden & 0.8742 & 0.9904 & 0.9915 & 0.9958 & 0.9984 & 1.0000 \\
\hline Nordihydroguaiaretic acid & 0.5502 & 0.9124 & 0.8709 & 0.9628 & 0.9982 & 0.9995 \\
\hline Pioglitazone & 0.7254 & 0.8356 & 0.8602 & 0.8940 & 0.9273 & 1.0000 \\
\hline Prochlorperazine & 0.7784 & 0.8932 & 0.8755 & 0.9241 & 0.9445 & 1.0000 \\
\hline Rosiglitazone & 0.6680 & 0.8705 & 0.8165 & 0.8958 & 0.9554 & 1.0000 \\
\hline Sirolimus & 0.8167 & 0.9301 & 0.9175 & 0.9541 & 0.9461 & 0.9995 \\
\hline Tanespimycin & 0.9635 & 0.9902 & 0.9828 & 0.9996 & 1.0000 & 1.0000 \\
\hline Thioridazine & 0.8140 & 0.8707 & 0.8770 & 0.8904 & 0.9323 & 1.0000 \\
\hline Tretinoin & 0.6016 & 0.9265 & 0.8383 & 0.9474 & 0.9650 & 1.0000 \\
\hline Trifluoperazine & 0.8371 & 0.9640 & 0.9546 & 0.9993 & 0.9986 & 0.9998 \\
\hline Valproic acid & 0.7202 & 0.9284 & 0.8749 & 0.9445 & 0.9646 & 1.0000 \\
\hline Vorinostat & 1.0000 & 1.0000 & 1.0000 & 1.0000 & 1.0000 & 1.0000 \\
\hline Wortmannin & 0.8912 & 0.9442 & 0.9478 & 0.9662 & 0.9707 & 0.9999 \\
\hline Trichostatin A & 0.9939 & 0.9998 & 0.9954 & 1.0000 & 1.0000 & 1.0000 \\
\hline Clozapine & 0.4935 & 0.9452 & 0.8869 & 0.9920 & 1.0000 & 1.0000 \\
\hline Estradiol & 0.5036 & 0.8451 & 0.8534 & 0.9344 & 0.9861 & 0.9998 \\
\hline Fluphenazine & 0.7064 & 0.8533 & 0.8909 & 0.8854 & 0.9359 & 1.0000 \\
\hline Troglitazone & 0.5646 & 0.7531 & 0.7173 & 0.8781 & 0.9596 & 1.0000 \\
\hline ALL & 0.7095 & 0.8645 & 0.8859 & 0.8948 & 0.9305 & 0.9999 \\
\hline
\end{tabular}

sion similarity search methods, multiple experiments were needed to measure co-expression between all pairs of genes and to perform a $t$-test for each gene. Therefore, we randomly selected 10 sub-experiment groups, which comprised 3 experiments, from each drug. We used all 10 experiments of each drug to infer the DCM signature and DEG signature. We tested our similarity search method using the combined score and previous methods for the 29 selected drugs listed in Table 2. Our novel similarity search method achieved very high performance compared to previous methods as shown in Figure 2. This means that, although the DCMs and
DEGs were identified from the target drug, the extracted gene signatures can be specific features for the target drug, which can be distinguished from other drugs, in addition to common features that are fairly representative properties for target drug response profiles.

\section{Construction of Drug Network}

After the performance measurement, we checked whether an identified gene signature can be effectively used to find novel associations between drugs and to find instances treated with the same drug. For this purpose, we made drug-drug 


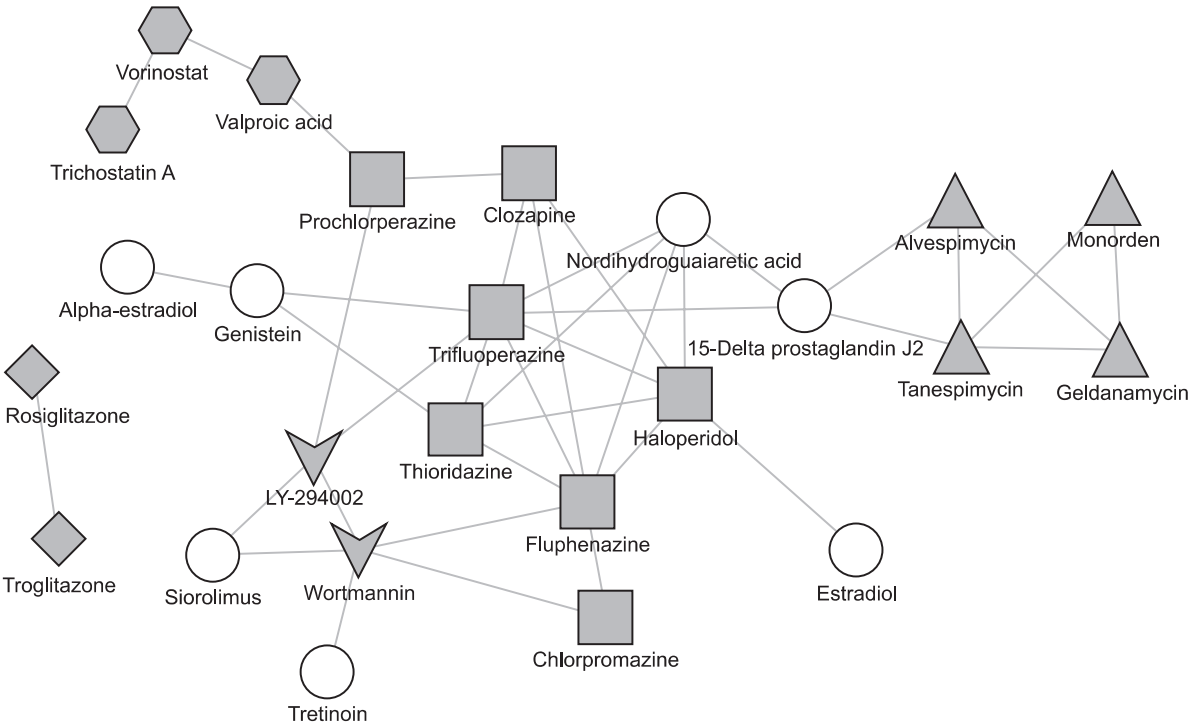

Figure 2. Predicted drug-drug network of selected 29 drugs. networks based on predicted similarity results among the 29 selected drugs. We calculated similarity scores based on the identified gene signature of each drug. The two similarity scores, $N \operatorname{Sim}_{\text {Combined }}^{\overrightarrow{A B}}$ and NSim Combined $_{\text {BA }}$, have different values because their query signatures are different from each other. Therefore, from the Equation (9), we defined the similarity score given to drugs $\mathrm{A}$ and $\mathrm{B}$ as in previous research [8]:

$$
N \operatorname{Sim}_{\text {Combined }}^{A B}=\frac{N \operatorname{Sim}_{\text {Combined }}^{\overrightarrow{A B}}+N \operatorname{Sim}_{\text {Combined }}^{\overrightarrow{B A}} .}{2}
$$

After the similarity measurement, we could get $\left(\begin{array}{c}29 \\ 2\end{array}\right)=406$ pairwise distances. To estimate the significance threshold for the distances, we used the empirical probability distribution function of these distances as in previous research [8]. In this research, we chose the significance threshold value as the upper bound of $10 \%$ of the empirical probability distribution. After filtering the pairwise distances using this threshold, we drew drug networks from the predicted similarities between drugs as illustrated in Figure 3. We grouped 25 drugs based on their target proteins. There were five target groups including 18 drugs. Those five groups of drugs are well clustered in this similarity network. Seven round-shaped drugs which have heterogeneous targets are scattered in the network. Some connections found in this similarity network between drugs for different known targets may imply the possibility of drug repositioning.

\section{Discussion}

We introduced a novel computational method for similarity search of drug response profiles. The main idea of our meth- od is the use of combined signatures of DCMs and DEGs. Integrating co-expressed gene modules can be more robust for various datasets with various numbers of samples and diverse conditions. The method was evaluated using searching experiments with data obtained from a profile library of cell lines treated with the same drug and identifying known drug groups that share the same pharmaceutical action. We demonstrated the high performance of our method with a 0.99 AUC score for searching the same drug by combined signature.

Drug repositioning is possible using the connection between drugs. Therefore, we predicted a drug-drug network to reveal the association between drugs. Although valproic acid is an anticonvulsant drug, it is associated with vorinostat and the same target protein. Thus, valproic acid can be a valuable drug for cutaneous T-cell lymphoma after clinical testing. Moreover, association between drugs can indicate a novel target. In our network, nordihydroguaiaretic acid targeting Acetyl-CoA acetyltransferase is similar to thioridazine, trifluoperazine, fluphenazine, and haloperidol targeting dopamine receptors. Therefore, we can infer that nordihydroguaiaretic acid has a novel target, dopamine receptors.

There were several limitations of our study. We used drug response profiles from the Connectivity Map. These profiles were obtained from only 5 cancer cell lines. It is limited in precisely measuring drug response profiles in relation to other diseases except several cancers. Therefore, it is necessary to investigate the drug response of disease-associated cell lines. For instance, to identify similar drugs for diabetes mellitus drugs, we need the drug response profiles in diabetes mellitus-associated cell lines, such as liver or adipocyte cell lines. Moreover, the number of samples for most drugs 
in drug response profiles is small. We only used the 29 selected drugs with more than 10 samples because DCMs need to calculate correlation in sufficient samples. Finally, we expected that response profiles using protein expression show better results than those using mRNA expression.

\section{Conflict of Interest}

No potential conflict of interest relevant to this article was reported.

\section{Acknowledgments}

This work was supported by the National Research Foundation of Korea grant funded by the Korea government (No. 2010-0028631). This research was supported by the Bio \& Medical Technology Development Program of the National Research Foundation funded by the Korean government (2012048758).

\section{References}

1. Qu XA, Rajpal DK. Applications of Connectivity Map in drug discovery and development. Drug Discov Today 2012;17(23-24):1289-98.

2. Engreitz JM, Morgan AA, Dudley JT, Chen R, Thathoo $\mathrm{R}$, Altman RB, et al. Content-based microarray search using differential expression profiles. BMC Bioinformatics 2010;11:603.

3. Le HS, Oltvai ZN, Bar-Joseph Z. Cross-species queries of large gene expression databases. Bioinformatics 2010;26(19):2416-23.

4. Fujibuchi W, Kiseleva L, Taniguchi T, Harada H, Horton P. CellMontage: similar expression profile search server. Bioinformatics 2007;23(22):3103-4.

5. Vazquez M, Nogales-Cadenas R, Arroyo J, Botias P, Garcia R, Carazo JM, et al. MARQ: an online tool to mine GEO for experiments with similar or opposite gene expression signatures. Nucleic Acids Res 2010;38(Web Server issue):W228-32.

6. Lamb J, Crawford ED, Peck D, Modell JW, Blat IC, Wrobel MJ, et al. The Connectivity Map: using geneexpression signatures to connect small molecules, genes, and disease. Science 2006;313(5795):1929-35.

7. Zhang SD, Gant TW. A simple and robust method for connecting small-molecule drugs using gene-expression signatures. BMC Bioinformatics 2008;9:258.

8. Iorio F, Bosotti R, Scacheri E, Belcastro V, Mithbaokar P, Ferriero R, et al. Discovery of drug mode of action and drug repositioning from transcriptional responses. Proc Natl Acad Sci U S A 2010;107(33):14621-6.

9. Shigemizu D, Hu Z, Hung JH, Huang CL, Wang Y, DeLisi C. Using functional signatures to identify repositioned drugs for breast, myelogenous leukemia and prostate cancer. PLoS Comput Biol 2012;8(2):e1002347.

10. Williams G. A searchable cross-platform gene expression database reveals connections between drug treatments and disease. BMC Genomics 2012;13:12.

11. Cheng J, Xie Q, Kumar V, Hurle M, Freudenberg JM, Yang L, et al. Evaluation of analytical methods for connectivity map data. Pac Symp Biocomput 2013:5-16.

12. Pacini C, Iorio F, Goncalves E, Iskar M, Klabunde T, Bork $\mathrm{P}$, et al. DvD: an R/Cytoscape pipeline for drug repurposing using public repositories of gene expression data. Bioinformatics 2013;29(1):132-4.

13. Yun T, Hwang T, Cha K, Yi GS. CLIC: clustering analysis of large microarray datasets with individual dimensionbased clustering. Nucleic Acids Res 2010;38(Web Server issue):W246-53.

14. Yun T, Yi GS. Biclustering for the comprehensive search of correlated gene expression patterns using clustered seed expansion. BMC Genomics 2013;14:144.

15. Lai Y, Wu B, Chen L, Zhao H. A statistical method for identifying differential gene-gene co-expression patterns. Bioinformatics 2004;20(17):3146-55.

16. Carter SL, Brechbuhler CM, Griffin M, Bond AT. Gene co-expression network topology provides a framework for molecular characterization of cellular state. Bioinformatics 2004;20(14):2242-50.

17. Kostka D, Spang R. Finding disease specific alterations in the co-expression of genes. Bioinformatics 2004;20 Suppl 1:i194-9.

18. Smyth GK, Michaud J, Scott HS. Use of within-array replicate spots for assessing differential expression in microarray experiments. Bioinformatics 2005;21(9):2067-75. 